- Highlights potentially fatal outcomes of poor management of acute severe dental pain.

- Raises questions about access to emergency dental services for patients.

- Discusses and advises approaches for management of acute irreversible pulpitis.

\title{
A life threatening event from poorly managed dental pain - a case report
}

\author{
R. W. J. Porter, ${ }^{1}$ N. J. Poyser ${ }^{2}$ and P. F. Briggs ${ }^{3}$
}

The history of a patient who suffered encephalopathy and coma is presented. A 25-year-old female consumed large quantities of cold water over several weeks, to control long-term dental pain. This eventually led to dilution hyponatraemia, followed by a seizure and encephalopathy. The patient made a good recovery after spending three days in neurological intensive care. Conventional endodontic therapy immediately resolved her symptoms following recovery from this lifethreatening episode. Prior to her admission the patient had experienced difficulties in gaining access to effective emergency dental care. Her problems could have been avoided if appropriate management had been provided sooner. The dental profession should be aware of the potential life-threatening risk when continued water consumption is used to control the long-term pulpitic pain. Primary care agencies should ensure that information on local emergency dental services is well disseminated to the population. Dental surgeons should be able to manage acute dental pain.

\section{INTRODUCTION}

Acute irreversible pulpitis (AIP) is an intensely painful condition ${ }^{1,2}$ which requires prompt intervention by dental professionals to provide appropriate treatment. ${ }^{3,4}$

Clinical signs and symptoms of any oral complaint should be carefully assessed, with the aid of appropriate special tests, to both confirm a diagnosis and treatment plan. Reaching a diagnosis can prove difficult. Squeezing emergency patients into busy schedules can reduce the time available for history taking, special tests and diagnosis. Dental professionals should ideally have adequate emergency care capacity. AIP has characteristics particular to it which

1,2Specialist Registrar in Restorative Dentistry, ${ }^{3}$ Consultant in Restorative Dentistry, Guys, Kings and St. Thomas' Dental Institute and St. George's Hospital Tooting, London, SW17 00T

${ }^{*}$ Correspondence to: Dr Richard Porter

Email: rich.porter7@ntlworld.com

\section{Refereed Paper}

Accepted 16 May 2006

DOI: $10.1038 /$ bdj.2007.144

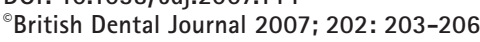

should facilitate reaching a diagnosis. ${ }^{1,5,6}$ There is good evidence supporting pulp extirpation or tooth extraction as appropriate treatment options. ${ }^{3,4}$

Occasionally, an obvious source of odontogenic pain may be overlooked. An incorrect diagnosis may lead to prolonged pain and suffering for the patient. In such circumstances it is likely the patient may seek further professional advice. They may also use analgesics and other coping mechanisms to relieve symptoms. Anecdotally the authors have found one such mechanism can be the use of cold substances held adjacent to the symptomatic tooth.

This clinical report highlights the history of an unregistered patient with acute irreversible pulpitis who presented to a number of state funded emergency dentists but received no effective treatment. Her mechanism for controlling her symptoms with cold water led to a life threatening event. For this patient these shortfalls could have proved fatal if it was not for the actions taken by her young children.

\section{CASE HISTORY}

A 25-year-old retail manager was brought by ambulance to the accident and emergency department at St. Helier Hospital, Croydon, UK following a collapse and seizure at her home.

The emergency services had been called by the patient's two young children who had found their mother in the midst of a seizure which lasted approximately 10 minutes. The patient exhibited generalised behavioural disturbances manifesting as agitation and aggression immediately following the seizure.

Upon arrival at the hospital the condition of the patient was scored at seven on the Glasgow Coma Scale (GCS). It remained at this level for six hours following the seizure. The patient was intubated and transferred to the intensive care unit under the neurology team at Atkinson Moreley Hospital, Wimbledon, UK. Magnetic resonance imaging revealed diffuse brain swelling. Some significant abnormalities were noted following routine blood screening (Table 1). 
A clear medical history was ascertained from relatives. The patient had no history of depression or mental illness. She drank alcohol socially and there was no evidence of illicit drug use.

A dental history obtained from the family revealed that the patient had been complaining for several weeks of severe dental/jaw pain, particularly in the right mandibular region. As emergency dentists had not been able to resolve her problems she was taking analgesics ( $2.6 \mathrm{~g}$ ibuprofen and $2.0 \mathrm{~g}$ paracetamol per day) and continuously holding ice cold water in her mouth which was then swallowed.

The neurologists diagnosed psychogenic polydipsia caused by the excessive water consumption. This led to dilution hyponatraemia and encephalopathy.

Under their care, she made a steady recovery and her serum sodium normalised after eight days. The patient was discharged with a short course of phenytoin.

\section{Dental management}

The patient was urgently seen on referral by the Department of Restorative Dentistry of St. George's Hospital. Unfortunately her dental pain was still present. She complained of a several week history of sharp pain initiated by hot fluids which outlasted removal of precipitating stimuli. Cold water held against the LR5 offered the most effective pain relief. The patient confirmed that she then swallowed the water and that this pattern had been repeated many times throughout the day. She had not been able to sleep normally for several weeks and she estimated that she consumed up to 10 litres of water each day. She complained of a mild dull ache affecting other quadrants.

\section{Previous dental management of the pain}

The patient had tried to register with a state funded dentist when her symptoms began. This proved problematic as she was advised that the dentists she contacted would only accept new patients on a self-funded basis. Eventually she did find and register with a state funded dentist who suggested that her pain was related to her jaw joint and would 'clear up in time'.

She was given a soft biteguard to wear and prescribed a course of antibiotics of which the details are not known. She also recollected that intra-oral radiographs

Table 1 Abnormal blood results obtained following admission to intensive care.

\begin{tabular}{l|l}
\hline Sodium & $123 \mathrm{mmol} / \mathrm{l}$ (normal range 135-145) \\
\hline Urine osmolality & $81 \mathrm{mmol} / \mathrm{kg}$ (normal range 100-1400) \\
\hline Serum osmolality & $261 \mathrm{mmol} / \mathrm{kg}$ (normal range 285-295)
\end{tabular}

were taken at this assessment. Due to the continuation of her problems, she attended two other practices, neither of whom offered an immediate appointment despite her description of the severity of the problem. She was seen for initial consultations at both these practices and was offered more antibiotics but received no 'hands on' treatment. The patient did not recollect receiving any advice with regards to alternatives for emergency dental care provision.

\section{Dental examination}

The patient had good mouth opening with no evidence of temporomandibular joint problems. Multiple amalgams were present in the posterior teeth. The LR5 was restored with a disto-occlusal amalgam. The tooth was mildly tender to percussion and acutely hypersensitive to heat. The pain outlasted the presence of hot stimuli and was causing severe spontaneous pain. Caries was also visible at UR6 and LL6.

Bitewings and periapical films confirmed that extensive caries was present beneath the restoration in LR5 (Fig. 1).

A diagnosis of acute irreversible pulpitis of LR5 and several other carious lesions was made. The LR5 was extirpated immediately under local anaesthesia. On review, this was found to have resolved her symptoms.

As the patient was now registered with a general dental practitioner, they were asked to complete the endodontic

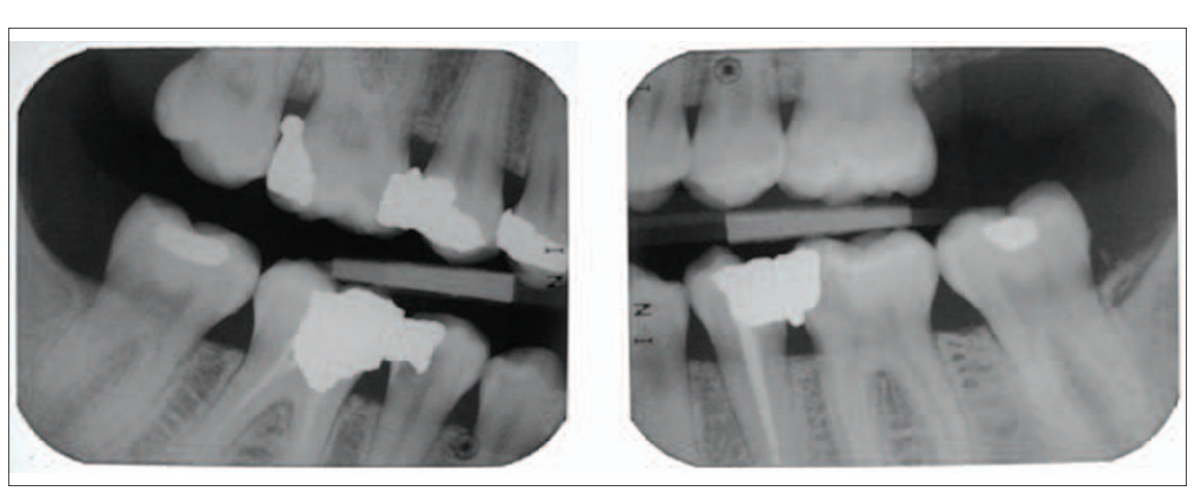

Fig. 1 Bitewings and periapical films confirmed that extensive caries was present beneath the restoration in LR5

treatment of LR5 and restorative treatment of UR6 and LL6. The patient was given appropriate advice on plaque control, diet and the use of daily fluoride.

\section{DISCUSSION}

Diagnosis of pain

Pain is cited as the primary reason for patients seeking emergency dental care. $^{7,8}$ The division of pulpitic symptoms into reversible or irreversible pulpitis has been described by several authors. ${ }^{1,5,6}$ Although localisation of pulpal pain can occasionally be difficult, irreversible pulpitis can usually be readily identified from the description of the pain. Bender ${ }^{1}$ suggested that AIP is commonly associated with the following pain characteristics:

- Spontaneous and severe typically described as an 'agonising throb'

- Thermal sensitivity - particularly to hot fluids

- Prolonged pain following the removal of an exacerbating factor

- Sleep interference.

The presence of a severe spontaneous dental pain should immediately make a dentist suspicious of an irreversible rather than reversible pulpitis. ${ }^{2}$

In retrospect, the symptoms described by this patient were likely to have related to acute irreversible pulpitis from the LR5. Either pulpal extirpation or extraction would have resolved the problem..$^{3,4}$

The poor localisation and lack of any

restoration in LR5 
direct visual evidence of cavitation in the LR5 may have complicated identification of the causative tooth.

Pulpal pain can be modulated by a number of factors which include inflammatory mediators and vascular changes (blood flow, volume and pressure).

The acute pain felt by patients with acute irreversible pulpitis is rarely the first episode of discomfort.9,10 Typically $60 \%$ of teeth become hypersensitive to thermal stimuli during pulpal damage. Fortunately for the dental profession $40 \%$ of pulps which become necrotic may do so without symptoms. ${ }^{6}$

Anecdotal evidence suggests that patients may find acute inflammatory conditions may be helped by holding cold liquids or objects adjacent to the affected site. This is likely to relate to vasoconstriction and a transient reduction of vascular flow within the pulp. This reduces pulpal pressure and modifies the sensory input from pain fibres. ${ }^{1}$ This could explain why the cold analgesic phenomenon, reported by the patient, was found to be more effective than the high doses of paracetemol and ibuprofen. In the authors' opinion, the patient's retrospective history was a classic example of AIP.

\section{PREVIOUS MANAGEMENT}

\section{Radiographic standards}

The patient remembered clearly that intra-oral radiographs were taken a month prior to presenting in our department. Despite the authors having made contact with the dentist it is not clear what these radiographs showed. A bitewing radiograph was necessary to show the caries in the LR5 as cavitation was not visible in the mouth. A number of possible reasons may have contributed to the situation where a radiograph was taken but did not reveal the extensive caries in LR5. It could be that the radiograph was not processed until the patient had left the surgery, or that, when developed, it was of suboptimal diagnostic value. It is likely caries would have been detected on a good quality radiograph and it is unclear why this was not pointed out to the patient when she returned for the fit of her bite raising appliance. It is also unclear why antibiotics were prescribed together with the bite raising appliance. Although there is good evidence that antibiotics are ineffective in the management of AIP, ${ }^{10-12}$ they are still commonly prescribed for this purpose. ${ }^{13}$

Unfortunately our attempts to obtain these radiographs were unsuccessful despite several phone calls and written correspondence.

\section{Problems with treating IP}

The patient reported that no invasive dental treatment was attempted by any of the dentists she saw when in acute pain. This may have been because the dentists were apprehensive about managing acute AIP or that the patient could not tolerate treatment.

Treating a patient in acute pain can be a stressful experience for the practitioner as well as the patient and prescribing oral antibiotics may often be seen as a convenient option.

Any practitioner who has attempted to anaesthetise a 'hot pulp' knows that this procedure itself may be time consuming and potentially problematic. Time implications may have been a factor in the management of this case.

It is disappointing that none of the dental surgeons attempted pulpal extirpation of the LR5 for this patient. She did not recollect any form of vitality testing taking place. Evidence would suggest that the use of electric pulp testing in dental practices is rare and indeed, this procedure can be unreliable. ${ }^{15}$

Although the caries may have not been clinically detectable without a radiograph her history alone would have suggested a diagnosis of AIP.

\section{The role of systemic antibiotics in the management of pulpitis}

Current evidence would not support the prescribing of systemic antibiotics for the management of AIP. Also they should be used rarely in the management of periapical infection. Their use should be reserved for control of spreading infection. ${ }^{3,4,11-13}$ Despite this, surgical intervention for pulpitis is not always the first treatment choice by the dental profession. ${ }^{11}$

Local anaesthetic in the treatment of AIP It is imperative that dentists receive training and are adequately skilled in the management of AIP. Conventional local anaesthetic techniques are sometimes unsuccessful in obtaining profound anaesthesia for endodontic procedures. A greater volume of local anaesthetic
(LA) agent can be used along with supplemental LA techniques.

Periodontal ligament injections (intraligamentary) and intraosseous injections may prove to be useful adjuncts. ${ }^{16-18}$ Once the pulp chamber is accessed, delivery of LA directly into the pulpal tissue usually provides profound anaesthesia predictably..$^{19}$ Intrapulpal injections usually have the disadvantage of being quite uncomfortable for the patient. In the authors' experience patients typically describe this discomfort as an acute but short lived 'burning sensation'.

Anecdotally, the authors also find the use of Articaine (Septodont, Saint-Muir, France) to be a useful adjunct to conventional lignocaine based LA where anaesthesia proves difficult, although there is little published evidence to support this. ${ }^{20}$

\section{Access to state funded emergency dental} services for the unregistered patient in the UK

Access to state funded dentistry remains a significant problem for many members of the UK population, varying with location and socio-economic status. ${ }^{21,22}$ The UK as a whole had a population to dentist ratio of 2,485 to 1 in 1994 . This was the second least favourable in the European Union. ${ }^{23}$

Studies have tried to measure the degree of inequality regarding availability of state funded dental practitioners in the UK. Some authors have hypothesised as to why inequality exists and how it may be addressed. As it stands however, the expansion of 'self-funded' healthcare continues and unregistered dental patients may be faced with a difficult search to find a state funded dental list to which they can be added.

In 1997 specific targets were set by the Department of Health to address this problem. NHS Direct was set up in the late 1990s. This provides UK patients with a phone service offering advice on health care. This service can direct patients to state funded practices or to appropriate emergency services. State funded dentists in the UK have a duty of care which states that a patient reporting pain should be seen in the practice or be informed about access to an emergency clinic within 24 hours of their complaint. The recently introduced new contract for NHS dental practitioners may help improve the problem of access 
to emergency dental care. Additionally, Primary Care Trusts are likely to target the set up of strategically placed, 'walk in' emergency dental access centres. These will offer treatment to unregistered patients.

\section{CONCLUSION}

This case history reports a rare but lifethreatening event that followed several weeks of severe pulpal pain. The patient was a fit 25-year-old mother of two children who tried unsuccessfully to get dental help to resolve her acute pain. After discharge from hospital a diagnosis of long-term acute irreversible pulpitis was made. During the acute phase of the patient's symptoms, despite contacting and having consultations with several dentists and being radiographed by one, her symptoms were not effectively treated. The circumstances of her management raise important questions including the level of access to high quality emergency dental care in the UK. Important issues highlighted in this case report relate to the organisers and providers of emergency dental services. Dentists must maintain practical skills to manage acute pulpal inflammation. Undergraduate and postgraduate teachers have an important role to ensure that the profession is adequately skilled to relieve such problems in an appropriate manner.
Continuing professional development (CPD) courses may provide opportunities for the dental profession to ensure skills in managing acute pulpitis are maintained. Currently many endodontic CPD courses have a bias towards the theory and mechanics of preparation, obturation and restoration using the 'latest gadgets'. As highlighted by this report, dentists being able to provide effective management of AIP is vitally important and the authors strongly advocate its inclusion in CPD courses.

1. Bender I. Pulpal Pain Diagnosis - A Review. J Endod 2000; 26: 175-179.

2. Cohen S, Liewehr F. Diagnostic procedures. In Cohen S, Burns R (Ed). Pathways of the pulp. $8^{\text {th }}$ ed. pp 26-29. St. Louis: Mosby, 2002.

3. Ogutembi B R, DeSchepper E J, Taylor T S et al. Post operative pain incidence related to the type of emergency treatment of symptomatic pulpitis. Oral Surg Oral Med Oral Pathol 1992; 73: 479-483.

4. Matthews R W, Peak J D, Scully C. The efficacy of management of acute dental pain. Br Dent J 1994; 176: 413-416.

5. Dummer P M, Hicks R, Huws D. Clinical signs and symptoms in pulp disease. Int Endod J 1980; 13: 27-35.

6. Michaelson $P$ L, Holland $G \mathrm{R}$. Is pulpitis painful? Int Endod J 2002; 35: 829-832.

7. O'Keefe E M. Pain in endodontic therapy: preliminary study. J Endod 1976; 2: 315-319.

8. Matthews R, Scully C, Porter K, Griffiths M. An analysis of conditions presenting to a dental hospital emergency clinic. Health Trends 1992; 24: $126-128$.

9. Santini A. The diagnosis, classification and treatment of acute pulpal pain by UK general dental practitioners: Results of a survey. Prim Dent Care 1996; 3: 24-27.

10. Nusstein J M, Beck M. Comparison of preoperative pain and medication use in emergency patients presenting with irreversible pulpitis or teeth with necrotic pulps. Oral Surg Oral Med Oral Pathol 2003: 96: 207-214.

11. Thomas D W, Satterthwaite J, Absi E G et al. Antibiotic prescription for acute dental conditions in the primary care setting. Br Dent J 1996: 181: 401-404.

12. Nagle $D$, Reader A, Beck M, Weaver J. Effect of systemic penicillin in untreated pulpitis. Oral Surg Oral Med Oral Pathol Oral Radiol Endod 2000; 90: 636-640.

13. Dailey Y M, Martin M V. Are antibiotics being used appropriately for emergency dental treatment? Br Dent J 2001; 191: 391-393.

14. Petersson K, Soderstrom C, Kiani-Anaraki M, Levy $G$. Evaluation of the ability of thermal and electrical tests to register pulp vitality. Endod Dent Traumatol 1999; 15: 6-16.

15. Palmer N A, Pealing $R$, Ireland R S, Martin M V. A study of therapeutic antibiotic prescribing in National Health Service general dental practice in England. Br Dent J 2000; 188: 554-558.

16. Walton R E. The periodontal ligament injection as a primary technique. J Endod 1990; 16: 62-66.

17. Childers M, Reader A, Nist R et al. Anasthetic efficacy of the periodontal ligament injection after an inferior alveolar nerve block. J Endod 1996; 22: 317-322.

18. Dunbar D, Reader A, Nist R et al. Anaesthetic efficacy of the intraosseous injection after an inferior alveolar nerve block. J Endod 1996; 22: 481-486.

19. Meechan J G. Supplementary routes to local anaesthesia. Int Endod J 2002; 35: 885-896.

20. Claffey E, Reader A, Nusstein J et al. Anesthetic efficacy of articaine for inferior alveolar nerve blocks in patients with irreversible pulpitis. J Endod 2004: 30: 568-571.

21. McGrath C, Bedi R, Dhawan N. Who has difficulty in registering with an NHS dentist? - A national survey. Br Dent J 2001; 191: 682-685.

22. Moles D R, Frost C, Grundy C. Inequalities in availability of National Health Service general dental practitioners in England and Wales. Br Dent J 2001; 190: 548-553.

23. Eaton K A, Widstroem E A, Renson C E. Changes in the numbers of dentists and dental caries levels in 12-year-olds in the countries of the European Union and economic area. J R Soc Health 1998; 118: 40-48. 